\title{
TRANSPLANTE HEPÁTICO: ASPECTOS HISTÓRICOS
}

\author{
Liver Transplantation: Historical Aspects
}

André Gusmão Cunha ${ }^{1,2,3}$, Jorge Luiz Andrade Bastos ${ }^{2,3}$, Roberto José Meyer-Nascimento ${ }^{1}$

\section{RESUMO}

Pouco mais de meio século se passou desde os trabalhos pioneiros de transplante experimental de fígado. Atualmente, o transplante hepático ortotópico é o tratamento de escolha para várias doenças hepáticas agudas e crônicas, que põem em risco a vida ou causam piora importante da qualidade de vida do paciente e que não são curáveis com outros tratamentos. Nesse meio século, a terapia de transplante hepático evoluiu com múltiplas etapas de desenvolvimento e afirmação.

Descritores: Transplante de Fígado; História da Medicina; Imunologia.

\section{Instituição:}

${ }^{1}$ Instituto de Ciências da Saúde, Universidade Federal da Bahia (ICSUFBA)

${ }^{2}$ Hospital Português, Serviço de Transplante Hepático, Salvador, Brazil

${ }^{3}$ Faculdade de Medicina da Bahia, Universidade Federal da Bahia (FMB-UFBA)

\section{Correspondência:}

Prof. André Gusmão Cunha

End.: Faculdade de Medicina da Universidade Federal da Bahia (UFBA), Unidade Acadêmica FMB, Curso de Medicina, Terreiro de Jesus, SN, Pelourinho, CEP 40000-000 - Salvador/BA, Brazil

Tel.: (71) 9159-6866

Fax: (71) 3256-8230

E-mail: andre_gusmao@ig.com.br

Recebido em: 11/02/2013

Aceito em: 18.03 .2013
Há pouco mais de uma geração, o tratamento de doenças hepáticas em estágio final era muito limitado, restrito à tentativa frustrada de aliviar os sintomas da piora clínica dos pacientes afetados. Estes acabariam passando pela insuficiência hepática e hipertensão portal, com hemorragias varicosas, ascites intratáveis, icterícia, peritonites, encefalopatia hepática e coagulopatia, culminando com a disfunção multissistêmica. É tido que a expansão do transplante de fígado transformou a hepatologia de uma disciplina meramente cerebral e acadêmica para uma especialidade ativa e intervencionista. ${ }^{1}$

A evolução da civilização humana tem nos presenteado com avanços inimagináveis em tempos anteriores. A medicina, parte ativa dessa civilização, é ambiente propício de desenvolvimento tecnológico surpreendente, onde "o que era inconcebível ontem e pouco viável hoje, muitas vezes torna-se a rotina de amanhã". ${ }^{2}$ 
Pouco mais de meio século se passou desde os trabalhos pioneiros de Welch com transplante experimental de fígado em modelos caninos. ${ }^{3-5}$ Atualmente, o transplante hepático ortotópico, que consiste na retirada de um fígado doente e sua substituição, na mesma localização anatômica, por um fígado, ou parte dele, saudável, procedente de um doador falecido ou vivo, ${ }^{6}$ é o tratamento de escolha para várias doenças hepáticas agudas e crônicas, que põem em risco a vida ou causam piora importante da qualidade de vida do paciente e que não são curáveis com outros tratamentos. ${ }^{6,7}$ Nesse meio século, a terapia de transplante hepático evoluiu com múltiplas etapas de desenvolvimento e afirmação.

O primeiro transplante de órgãos sólidos com êxito aconteceu em 1954, com um transplante de rim entre irmãos gêmeos idênticos, realizado por Murray, o que lhe valeu reconhecimento com um Nobel em Medicina. ${ }^{8}$ Porém, enquanto as primeiras abordagens para a imunossupressão permitiram o florescimento do transplante renal, os esforços iniciais do transplante de fígado estagnaram, mesmo com as evidências em animais que sugeriam ser o fígado um órgão imunologicamente privilegiado. ${ }^{1,9-12}$

Surgiram as primeiras tentativas de transplante hepático ortotópico em humanos, organizadas em ensaios clínicos, e com elas os primeiros insucessos..$^{13} \mathrm{O}$ primeiro receptor, operado por Starzl, sangrou até a morte na mesa cirúrgica. No ano seguinte, mais cinco tentativas foram realizadas por Starzl e mais duas em outros serviços, porém nenhum dos receptores sobreviveu mais de 23 dias. Com essas primeiras mortes, o transplante de fígado parecia ter encontrado obstáculos intransponíveis, como a má função inicial do enxerto resultante de lesão isquêmica e opções limitadas de imunossupressão (azatioprina e prednisona), o que levavam à coagulopatia grave, infecção e falência de múltiplos órgãos. ${ }^{1}$

A introdução clínica, em 1966, do soro antilinfócito fez Starzl ousar tentar mais uma vez e, em 1967, ele realizou o primeiro transplante de fígado bem sucedido em humanos, com sobrevida de 13 meses. ${ }^{14}$ Em 1968, Calne realizou o primeiro transplante de fígado humano na Europa. ${ }^{1}$

A evolução do conhecimento da fisiologia e anatomia cirúrgica do fígado, aliado ao desenvolvimento da preservação do fígado extraído, trouxe esperança ao procedimento e produziu as primeiras sobrevidas acima de um mês. ${ }^{15}$ Ainda assim, a sobrevida média dos transplantados hepáticos era menor que $50 \%$ em um ano. ${ }^{2}$ Devido aos altos riscos e resultados pobres, o transplante de fígado ficava reservado como um ato derradeiro e heroico para pacientes sem outras opções.
Mesmo com o reconhecimento da morte encefálica, ${ }^{16}$ que possibilitou a captação de fígados mais preservados e levou à melhora da função hepática inicial, a mortalidade permanecia alta. ${ }^{1}$

A conquista seguinte - um melhor controle sobre a imunossupressão-trouxe o ava nço dalonga sobrevida no póstransplante. A descoberta da atividade imunossupressora da ciclosporina por Borel, ${ }^{17,18}$ seguida pelo surgimento do tacrolimo, ${ }^{19-21}$ colocou o transplante hepático sob nova perspectiva, permitindo o desenvolvimento de programas de transplante por todo o mundo. A taxa de sobrevida em um ano era de pelo menos $70 \%,{ }^{22}$ com o advento dos primeiros pacientes a sobreviverem por mais de uma década. ${ }^{23}$ Esse foi o ponto de inflexão da sobrevida, que fez o transplante de fígado ser considerado como aceitável, aplicável clinicamente e salvador de vidas em $1983 .^{24}$

Outros avanços tornaram o transplante hepático mais seguro, organizado, rápido e reprodutível. A técnica operatória evoluiu, com alterações importantes. ${ }^{25} \mathrm{~A}$ circulação extracorpórea veno-venosa foi substituída pela técnica de preservação da veia cava retrohepática "piggyback". A incidência de complicações biliares foi reduzida com a melhor preservação do tecido periductal.

Avanços na avaliação e tratamento da coagulopatia com os conhecimentos obtidos pelos trabalhos de Kang, ${ }^{26,27}$ e aprimoramento da técnica operatória diminuíram a necessidade transfusional e suas decorrentes complicações e implicações, além de melhorias na captação, preservação e alocação de órgãos. ${ }^{21}$

A aplicação do transplante de fígado expandiu-se para incluir favoravelmente pacientes mais saudáveis no início da doença hepática, o que melhorou ainda mais seus resultados. Tal expansão de indicações chegou a ponto de incluir antigas contraindicações, como hepatite B, etilismo crônico, carcinoma hepatocelular, trombose de veia porta, idade avançada, dentre outras. Atualmente o transplante de fígado exibe taxas de sobrevida acima de $85 \%$ no primeiro ano e acima de $70 \%$ em cinco anos, ${ }^{1}$ com custos altos, mas que equivalem às reinternações frequentes de pacientes cirróticos descompensados. ${ }^{2}$

Ainda assim, novos problemas são sempre motivações para novos avanços. "O transplante de órgãos é vítima de seu próprio sucesso" ${ }^{28}$ o que tornou a falta de órgãos disponíveis o problema a ser resolvido. Doação intervivos, "splitliver" e o transplante dominó são exemplos de técnicas desenvolvidas para ajudar a solucionar as consequências dessa escassez, além do uso de fígados doados considerados marginais. ${ }^{1,25}$ 


\section{ABSTRACT}

Little more than half a century has passed since the pioneering works on experimental liver transplantation. Currently, orthotopic liver transplantation is the treatment of choice for various acute and chronic liver diseases, which endanger life or cause significant deterioration of the quality of life of patients that are not curable with other treatments. In half a century, the therapy for liver transplantation has evolved with multiple stages of development and affirmation.

Keywords: Liver Transplantation; History of Medicine; Immunology.

\section{AGRADECIMENTOS}

O autor agradece ao Prof. Raymundo Paraná, por sua sugestão e incentivo para a publicação deste artigo, e aos membros do Serviço de Transplante Hepático do Hospital Português em Salvador, incansáveis em sua luta para acolher e tratar bem os doentes.

\section{REFERÊNCIAS}

1. Dienstag J, Cosimi A. Liver Transplantation - A Vision Realized. N Engl J Med [Internet]. 2012 Oct 18 [cited 2012 Oct 20];367(16):14835. Available from: http://www.ncbi.nlm.nih.gov/pubmed/23075174

2. Starzl TE, Iwatsuki S, Van Thiel DH, Gartner JC, Zitelli BJ, Malatack JJ, et al. Evolution of Liver Transplantation. Hepatology. 1982;2:61436.

3. Moore FD, Smith LL, Burnap TK, D DF, Dammin GJ, Gruber UF, et al. One-stage homotransplantation of the liver following total hepatectomy in dogs. Transpl Bull. 1959;6(1):103-7.

4. Starzl TE, Kaupp Jr HA, Brock DR, Linman JW. Studies on the rejection of the transplanted homologous dog liver. Surg Gynecol Obs. 1961;112:135-44.

5. Welch CS. A note on transplantation of the whole liver in dogs. Transpl Bull. 1955;2:54-5.

6. Prados E, Cuervas-Mons V. Transplante hepático. Indicaciones y contraindicaciones generales. Elección del momento. In: Berenguer J, Bruguera M, García M, Sáez LR, editors. Tratamiento de las Enfermedades Hepáticas y Biliares Asociación Española para el Estudio del Hígado. Madrid: Elba; 2005. p. 411-23.

7. Keeffe EB. Liver Transplantation: Current Status and Novel Approaches to Liver Replacement. Gastroenterology [Internet]. 2001 Feb [cited 2012 Oct 31];120(3):749-62. Available from: http:// linkinghub.elsevier.com/retrieve/pii/S0016508501630026
8. Snyder A. Joseph E Murray. Lancet. 2013;381(9861):110.

9. Kamada N, Davies H ff. S, Roser B. Reversal of Transplantation Immunity by Liver Grafting. Nature. 1981;292:840-2.

10. Kamada N. The immunology of experimental liver transplantation in the rat. Immunology [Internet]. 1985 Jul;55(3):369-89. Available from: http://www.pubmedcentral.nih.gov/articlerender.fcgi?arti $\mathrm{d}=1453645 \&$ tool $=$ pmcentrez\&rendertype $=$ abstract

11. Orlando G, Soker S, Wood K. Operational Tolerance after Liver Transplantation. J Hepatol. 2009;50(6):1247-57.

12. Thomson AW, Knolle $\mathrm{P}$ a. Antigen-presenting cell function in the tolerogenic liver environment. Nat Rev Immunol [Internet]. Nature Publishing Group; 2010 Nov [cited 2012 Nov 18];10(11):753-66. Available from: http://www.ncbi.nlm.nih. gov/pubmed/20972472

13. Starzl TE, Marchioro TL, Von Kaulla KN, Hermann G, Brittain RS, Waddell WR. HOMOTRANSPLANTATION OF THE LIVER IN HUMANS. Surg Gynecol Obs. 1963;117:659-76.

14. Starzl TE, Fung JJ. Themes of liver transplantation. [Internet]. Hepatology (Baltimore, Md.). 2010. p. 1869-84. Available from: http://www.ncbi.nlm.nih.gov/pubmed/20235333

15. Starzl TE, Groth CG, Brettschneider L, Penn I, Fulginiti VA, Moon JB, et al. Orthotopic Homotransplantation of the Human Liver. Ann Surg. 1968;168(3):392-414. 
16. Beecher HK. Ethical problems created by the hopelessly unconscious patient. [Internet]. The New England journal of medicine. 1968. p. 1425-30. Available from: http://www.ncbi.nlm. nih.gov/pubmed/5652626

17. Borel JF, Feurer C, Magnée C, Stähelin H. Effects of the new antilymphocytic peptide cyclosporin $\mathrm{A}$ in animals. Immunology [Internet]. 1977 Jun;32(6):1017-25. Available from: http://www. pubmedcentral.nih.gov/articlerender.fcgi?artid $=1445439 \&$ tool $=p$ mcentrez\&rendertype $=$ abstract

18. Calne RY, Rolles K, White DJG, Thiru S, Evans DB, McMaster P, et al. Cyclosporin A Initially as the Only Immunosuppressant in 34 Recipients of Cadaveric Organs: 32 Kidneys, 2 Pancreases, and 2 Livers. Lancet. 1979;2(November):1033-6.

19. McMaster P, Buist L. FK 506 in transplantation. Transpl Proc. 1993;25(4):2684-5.

20. McMaster P, Gunson B, Min X, Afonso R, Bastos J. Liver transplantation: changing goals in immunosuppression. Transpl Proc. 1998;30(5):1819-21.

21. Starzl TE, Demetris AJ, Van Thiel D. Liver transplantation. N Engl J Med. 1989;321:1014-22.

22. Iwatsuki S, Starzl Te, Todo S, Gordon Rd, Esquivel Co, Tzakis Ag, et al. Experience in 1,000 liver transplants under cyclosporine-steroid therapy: a survival report. Transpl Proc. 1988;20(Suppl 1):498-504.
23. Starzl TE. History of Liver and Other Splanchnic Organ Transplantation. In: Busuttil RW, Klintmalm GB, editors. Transplantation of the liver. 1996. p. 3-22.

24.National Institutes Of Health - Consensus Development Conference Statement Hepatology. 1984;4(suppl):107S$110 \mathrm{~S}$

25. Adam R, McMaster P, O'Grady JG, Castaing D, Klempnauer $\mathrm{JL}$, Jamieson $\mathrm{N}$, et al. Evolution of liver transplantation in Europe: report of the European Liver Transplant Registry. Liver Transpl [Internet]. 2003 Dec [cited 2013 Jan 20];9(12):1231-43. Available from: http://www.ncbi.nlm.nih. gov/pubmed/14625822

26. Kang Y, Lewis J, Navalgund A, Russe M, Bontempo F, iren L, et al. Epsilon-aminocaproic acid for treatment of fibrinolysis during liver transplantation. Anesthesiology. 1987;66:76673.

27. Kang Y. Coagulopathies in Hepatic Disease. Liver Transplant. 2000;6(4 (Suppl 1)):72-5.

28. Pollard S. Nephrology Dialysis Transplantation The impact of state legislation on organ donation - results of a US pilot scheme. 1997;2510-1. 